\title{
Étude de la Concurrence sur le Marché de la Téléphonie Mobile au Maroc: Revue de Littérature
}

\author{
Zineb Hassani Allaf \\ Etudiante-Chercheuse au Centre des Études Doctorales en Économie et \\ Gestion, Faculté des Sciences Juridiques, Économiques et Sociales de \\ Tanger, Université Abdelmalek Essaadi, Morocco
}

\section{Mohamed Drissi Bakhkhat}

Enseignant Chercheur, Faculté des Sciences Juridiques, Économiques et Sociales de Tanger, Université Abdelmalek Essaadi, Morocco

Doi:10.19044/esj.2019.v15n4p114～㞷L:http://dx.doi.org/10.19044/esj.2019.v15n4p114

\section{Résumé}

Le secteur de la téléphonie mobile est un secteur en plein essor, qui connaît des mutations majeures depuis la libéralisation et la numérisation des télécommunications. Pourtant, la téléphonie mobile représente au sein de l'industrie des télécommunications le secteur qui a connu la plus forte croissance. La concurrence au sein de ce secteur a fortement évolué pour aboutir à une forme oligopolistique. Avant la téléphonie mobile, le marché des télécommunications était essentiellement composé de services voix fixes fournis par un seul opérateur (monopole public). Cet article propose une revue de littérature dont l'objectif est de développer une analyse, menée par la littérature économique internationale, théorique et empirique sur la concurrence des télécommunications, plus précisément sur le segment mobile.

Mots-clés: Téléphonie mobile, libéralisation, privatisation, régulation concurrence, économie industrielle, modèle SCP. 


\title{
Study of Competition in the Mobile Telephony Market in Morocco: A Literature Review
}

\author{
Zineb Hassani Allaf \\ Etudiante-Chercheuse au Centre des Études Doctorales en Économie et \\ Gestion, Faculté des Sciences Juridiques, Économiques et Sociales de \\ Tanger, Université Abdelmalek Essaadi, Morocco
}

\section{Mohamed Drissi Bakhkhat}

Enseignant Chercheur, Faculté des Sciences Juridiques, Économiques et Sociales de Tanger, Université Abdelmalek Essaadi, Morocco

\begin{abstract}
The mobile sector is a booming sector that has undergone major changes since the liberalization and digitization of telecommunications. Yet, mobile telephony represents the fastest growing sector in the telecommunications industry. Competition within this sector has changed significantly to an oligopolistic form. Before the advent of mobile telephony, the telecommunications market consisted mainly of fixed voice services provided by a single operator (public monopoly). This paper focuses on developing an analysis which was conducted by the international economic literature on theoretical and empirical competition of telecommunications, more precisely on the mobile segment.
\end{abstract}

Keywords: Mobile telephony, liberalization, privatization, regulation, competition, industrial organisaion, SCP model.

\section{Introduction}

Depuis son ouverture en privé, le marché de la téléphonie mobile au Maroc a connu un fort dynamisme qui a dépassé les prévisions et a surpris aussi bien le Gouvernement que les opérateurs eux-mêmes. Cette dynamique est rendue possible avec l'arrivée de nouveaux concurrents en l'occurrence Médi Telecom/Orange et Wana Corporate/Inwi, ces derniers entrants ont contribué à l'émergence d'une nouvelle configuration de l'industrie de la téléphonie mobile en passant d'une structure monopolistique à une structure qui regroupe trois opérateurs. Une nouvelle dynamique concurrentielle est apparue, son analyse doit nous permettre d'évaluer le potentiel de développement de cette industrie au Maroc. 
Actuellement, le marché de la téléphonie mobile répond aux caractéristiques d'un oligopole, un petit nombre d'opérateurs desservant l'ensemble d'un marché très dense où chaque concurrent prend ses décisions en tenant en compte les décisions des autres opérateurs. Dès lors, ces opérateurs deviennent des concurrents directs et indépendants, livrant une concurrence acharnée. Par ailleurs, pour sortir gagnant du jeu concurrentiel, les opérateurs à travers leurs stratégies concurrentielles exercent d'importantes influences sur le comportement de leurs concurrents et sur la structure du secteur ( Hassani Allaf \& Drissi Bakhkhat 2018).

A travers d'importantes contributions théoriques et empiriques à la compréhension de ce type d'analyse, le modèle SCP (StructureComportement-Performance) nous parait fort utile. D'abord pour comprendre la structure et les comportements des entreprises en place et pour prendre en compte ensuite de toute la dimension sectorielle et institutionnelle de la bataille concurrentielle sur ce marché dans lequel l'Etat joue un rôle important.

Aussi, le marché de la téléphonie mobile est un terrain propice qui nous permettra d'appréhender les différentes dynamiques qui évoluent en parallèle dans l'environnement institutionnel des télécommunications à travers la libéralisation, la privatisation et la régulation du secteur. Tous ces éléments vont nous aider à comprendre les principales mutations de ce secteur..

\section{Libéralisation des télécommunications}

Un des enjeux majeurs du contexte d'évolution des opérateurs de télécommunications est leur confrontation à un environnement ouvert à la libéralisation dès les années 1990. Ces entreprises sont contraintes de se moderniser et d'innover afin d'assurer leur continuité sur un secteur devenu concurrentiel.

La justification de la libéralisation du secteur de télécommunications tient de la volonté de promouvoir le développement des affaires par le libre accès au marché. La libéralisation permet ainsi d'instaurer la concurrence et met fin à une situation de monopole. Au-delà de l'instauration de la concurrence, elle permet de stimuler l'activité des acteurs économiques et par conséquent de favoriser l'efficacité des entreprises évoluant sur le marché. Cette efficacité se manifeste par une nouvelle dynamique du marché. Certaines firmes vont se positionner favorablement sur le marché pendant que d'autres peuvent avoir des difficultés.

O.Boylaud et G.Nicoleti (2001) ont dégagé, dans une étude de l'OCDE, l'impact de la libéralisation dans le secteur des télécommunications. Les auteurs ont montré en effet sur la base des données de 24 pays de l'OCDE sur la période de 1991 à 1997 que la libéralisation a entraîné une augmentation de la productivité, une baisse des prix et une amélioration de la qualité de service. 
A partir de 1984 et étant inspiré par la nouvelle approche des télécommunications définie par l'union Européenne, le Maroc engage une mise en conformité du dispositif juridique et institutionnel en vue de parvenir à moyen terme une libéralisation totale du secteur des télécommunications. Une telle réforme implique l'application de nouvelles dispositions réglementaires qui doivent préparer l'ouverture progressive du secteur à la concurrence. Cette approche repose principalement sur trois axes : D'abord, une séparation des activités postales et de télécommunications en deux entités distinctes. Ensuite, disparition des monopoles publics à travers l'entrée sur le marché de nouveaux opérateurs. Et enfin, installation d'une agence autonome et indépendante de régulation pour surveiller, contrôler et corriger les pratiques anticoncurrentielles (Mezouaghi, 2005).

Cette réforme sectorielle a marqué l'histoire de la libéralisation de ce secteur au Maroc et depuis cette date, des transformations s'accélèrent avec le vote de la nouvelle Loi de télécommunications sous la pression notamment économique et technologique. La Loi $\mathrm{N}^{\circ}$ 24-96 relative à la poste et aux télécommunications et ses Décrets d'application adoptent la libéralisation du secteur et ainsi la fin du monopole au Maroc. Comme résultat direct, l'année 1998 marquait deux réalisations historiques, la scission de l'Office National des Postes et des Télécommunications (ONPT) en deux entités distinctes, Itissalat al-Maghrib (IAM) et Barid al-Maghrib (BAM) et la création d'une agence de réglementation autonome, l'Agence Nationale de Réglementation des Télécommunications (ANRT). Deux opérations exceptionnelles vont marquer le paysage tant sectoriel que macroéconomique et politique, la première est l'attribution de la seconde licence GSM en juin 1999, la deuxième est la privatisation partielle de l'opérateur historique en décembre 2000 (Hibou \& Tozy, 2002).

Dans le respect des règles juridiques établies, de la réforme des télécommunications et au regard des recettes financières générées, l'expérience de la libéralisation du marché de la téléphonie mobile au Maroc a été perçue comme une réussite.

\section{Privatisation des télécommunications}

La privatisation est définie comme un processus qui peut conduire à un transfert de tout ou en partie de la fonction de décision à des agents privés, et aussi, permettre de transférer intégralement les fonctions de décision et de contrôle à différents acteurs non publics, en privilégiant une séparation fonctionnelle plus nette comparativement au contexte public (Chatelin, 2002).

La privatisation s'oppose à la nationalisation qui suit un processus contraire à savoir transférer une propriété privée dans l'emprise de la propriété publique. Le phénomène de privatisation suit ou précède la libération d'un secteur dans lequel des opérateurs publics évoluaient. Elle impacte le modèle 
organisationnel de l'entreprise publique et vise entre autres la performance. Ainsi, une corrélation est faite entre privatisation et performance.

La privatisation a fait l'objet de multiples analyses qui proposent deux déterminants de la performance : la nature de la propriété et la concurrence (Catelin \& Chatelin, 2001).

Les privatisations ont amélioré globalement les performances des entreprises de l'industrie des télécommunications. Dans ces industries, la privatisation des opérateurs historiques n'a jamais été totale. Cela se justifie par le besoin et le souci de contrôler un secteur généralement stratégique. Cet Etat devient la plupart du temps en plus d'être toujours dans son rôle de production de règles (législation et réglementation) actionnaire et régulateur. Toutefois, la régulation est confiée à une entité qui bien que publique, détient certaines prérogatives telles que l'autonomie et l'indépendance.

La privatisation du secteur des télécommunications au Maroc s'est accompagnée de l'arrivée de grands opérateurs internationaux, le groupe Telefonica, un opérateur global côté dans les principales bourses du monde et première multinationale espagnole, Portugal Telecom qui est la première compagnie portugaise de télécommunications, présente sur les marchés financiers mondiaux et le français Vivendi qui entre pour $35 \%$ dans l'opérateur historique marocain. Ces opérateurs concrétisent surtout une stratégie d'ouverture à la concurrence et d'adoption de méthodes de gestion privées (Hibou \& Tozy, 2002 ; ANRT, Rapport annuel, 2001).

\section{Régulation des télécommunications}

Les marchés de télécommunications lorsqu'elles sont ouvertes à la concurrence, généralement de type oligopolistique, sont fortement réglementées et régulées. La régulation vise à faire jouer pleinement les règles et mécanismes de la concurrence. Ce mécanisme permet une institutionnalisation à travers la mise en œuvre de règles et de normes visant une cohérence des politiques publiques dans le cadre de l'encadrement d'une industrie (Smith, 2011). Aussi, la régulation des industries implique plusieurs échelles d'action publique, collective et privée et comporte des formes institutionnelles d'adhésion au régime international. Ceci confère à la régulation une dimension internationale par le fait d'organisations mondiales telles que l'Union Internationale des Télécommunications pour ce qui concerne les Technologies de l'information et de la communication et les télécommunications. Toutefois, une déclinaison des mécanismes de régulation dans des ensembles sous régionaux et nationaux s'opère pour tenir compte des spécificités régionales, nationales et locales.

Pour le Maroc, l'Agence Nationale de Réglementation des Télécommunications (ANRT) a un champ d'action varié entre mission de participation à la préparation des actes législatifs et réglementaires régissant 
le secteur des télécommunications et des attributions nécessaires pour élaborer de sa propre initiative ou à la demande de l'autorité gouvernementale compétente, les propositions visant à adapter le cadre juridique, économique et sécuritaire dans lequel s'exercent les activités de télécommunications. Des avancées importantes ont été réalisées dans ce sens, il y'a lieu de citer d'abord, les apports de la Loi $\mathrm{N}^{\circ}$ 55-01 qui modifie celle $\mathrm{N}^{\circ}$ 24-96 en matière de régulation des litiges relatifs au respect des articles 6,7 et 10 de la Loi $\mathrm{N}^{\circ} 06-$ 99 sur la liberté des prix et de la concurrence (Conseil de la concurrence, 2011). Ces nouvelles missions de l'ANRT ont permis de renforcer la régulation sectorielle des télécommunications au Maroc. Ensuite et dans la même lignée, la publication de la première note d'orientation générale pour la libéralisation du secteur des télécommunications (Hibou \& Tozy, 2002).

Avec l'ouverture à la concurrence, la privatisation et les changements intervenus au niveau institutionnel, la question de la concurrence et du positionnement des opérateurs du marché de la téléphonie mobile se pose avec acuité.

\section{Concurrence sur le marché de la téléphonie mobile}

Les avantages liés à la concurrence dans le secteur des télécommunications sont prouvés à travers de nombreux études. Les auteurs D.Lien et Y.Peng (2001) ont évalué l'efficacité du développement de 24 pays de l'OCDE et ont affirmé que les pays qui adoptent la concurrence dans leur industrie ont un rendement plus élevé que les pays dominés par le monopole (Lien \& Peng, 2001). Dans le même sens, S.Wallsten (2005), en se basant sur des données statistiques durant la période (1990-2000) de 30 pays africains et latino-américains, a indiqué l'influence et le poids de la concurrence sur la performance de l'industrie des télécommunications (Wallsten, 2001).

Pour l'analyse économique de la concurrence sur le marché de la téléphonie mobile, il est important de comprendre la nature de la concurrence et la typologie de marché étudié. Dans notre cas, le marché ciblé est un marché dense où le nombre des offreurs est limité, le nombre des demandeurs est élevé et où la concurrence entre acteurs est imparfaite. Il s'agit d'une étude portant surtout sur l'économie industrielle. Avant de présenter les études empiriques qui ont abordé ce sujet, il est utile de consacrer quelques lignes à l'état théorique de cette discipline.

\subsection{Cadre théorique}

L'économie industrielle est une branche de la Microéconomie qui analyse des comportements et stratégies des entreprises et de la concurrence sur les marchés, une étude des structures des marchés pour une analyse des performances (Carlton \& Perloff, 1988). Au cours de son évolution, la structuration de l'économie industrielle a connu des phases distinctes mais qui 
se complètent. Pour bien décrire les principaux apports d'auteurs, un ouvrage de référence est celui de F.M.Scherer, l'auteur signale dans son étude les imperfections dans le fonctionnement des marchés (Scherer \& Ross, 1970). Sur le plan de l'analyse théorique de ces imperfections, figure évidemment l'héritage des économistes de la concurrence imparfaite, parmi lesquels on peut citer A.A.Cournot, J.Dupuit,J.Bertrand et E.Chamberlain. Mais c'est surtout sur le plan des travaux empiriques que l'économie industrielle de la première génération a prospéré, à travers le paradigme $\mathrm{SCP}$, introduit par l'école de Harvard (Mason, 1957 ; Bain, 1959) développé par l'école de Chicago (Stigler, 1964 ; Demsetz, 1973) et par la suite amélioré par l'apport des auteurs de la Nouvelle Economie Industrielle (Schmalensee, 1980 ; Jacquemin, 1986 ; Tirole \& Laffont, 2000).

Après une brève présentation des fondements du modèle, nous essayons de montrer sa portée et ses limites.

\subsubsection{Le paradigme SCP}

Le paradigme Structure-Comportement-Performance (SCP) est considéré comme un schéma de base de l'économie industrielle. D'après ce paradigme, la structure du marché (nature de l'offre et de la demande, caractéristiques des produits, conditions d'entrée et la concentration entre autres) détermine le comportement des firmes (ce que font les entreprises, et la manière dont elles le font), cela inclut les stratégies de positionnement, des prix, les pratiques de collusion entre acteurs, les fusions et les acquisitions en matière d'investissement, de publicité. Tel comportement influence de sa part leurs performances, en général mesurées sur le plan de la rentabilité, de la croissance, de l'efficacité, du progrès technique.

Le modèle SCP a été développé au début par l'école de Harvard, il était considéré l'outil le plus performant pour l'analyse des industries et des comportements concurrentiels jusqu'aux années 1960-1970. Selon le paradigme traditionnel de ce modèle, les caractéristiques structurelles du marché orientent les comportements des entreprises qui impactent leurs performances. Mais dans les années 1970, le modèle a connu une deuxième vague de modification par l'ajout d'autres liens de rétroaction à cette causalité univoque, l'école de Chicago (Stigler, 1964 ; Demsetz, 1973), a remis en cause le paradigme SCP. Pour cette école, le comportement des firmes est le facteur explicatif déterminant de la performance du marché et cette dernière influence la structure (C P S). Le mécanisme régulateur est la libre concurrence entre les entreprises donc peu d'actions sur la structure. Mais l'école de Chicago ne disposait pas d'outils nécessaires pour décrire les interactions stratégiques. L'arrivée de la nouvelle Economie Industrielle à partir des années 1980, a donné une relecture stratégique à l'économie industrielle en s'appuyant sur la 
théorie des jeux (Tirole) et les tests empiriques, ainsi le paradigme SCP s'est orienté vers une relation inverse telle que $\mathrm{P}$ influence $\mathrm{C}$ et $\mathrm{S}$.

De façon plus générale, le paradigme conditionne l'état du marché par son environnement global, il est à l'origine des résultats importants pour comprendre le comportement des firmes sur les marchés, mais cette approche présente plusieurs faiblesses.

\subsubsection{Les limites du modèle SCP}

D'après ce paradigme, la plupart des industries ont des structures intermédiaires entre la concurrence parfaite et le monopole. Il s'agit donc de savoir dans quelle mesure le niveau des prix et celui des profits demeurent proches de ceux de la concurrence ou, au contraire s'approchent de ceux du monopole. G.S.Stigler (1964) a montré que plus le nombre d'entreprise est élevé, plus il est difficile de repérer les comportements anticoncurrentiels et de les sanctionner. D'autres caractéristiques du marché aggravent également cette difficulté, comme l'innovation, le taux de croissance du marché, l'existence de fortes relations de clientèle ou encore la différenciation des produits.

Les limites du modèle proviennent également du fait que la chaîne de causalité entre S-C-P est univoque : Elle part des structures de marché pour aboutir à la rentabilité. Or, ceci peut être contesté en considérant que les profits des firmes exercent également une influence sur les structures des marchés. De même, la négligence des comportements n'est pas raisonnable, les firmes peuvent avoir des comportements stratégiques et donc peuvent par leur action influencer les structures des marchés. Ensuite, les études SCP sont basées sur des données transversales alors que la structure et la performance change d'une industrie à l'autre, et surtout pour les taux de concentration souvent utilisés comme indice de mesure du pouvoir de marché, ces taux ne sont pas les plus performants pour les marchés économiques pertinents.

En général, les chercheurs de l'approche SCP supposent que le pouvoir de marché ou la performance est facilement mesurable et sacrifient plus d'importance à la relation entre la performance et la structure, alors que ces mesures ne reflètent ni la performance ni la structure. D'autres données et techniques modernes sont apparus et qui sont plus adaptées pour mesurer le degré du pouvoir de marché. En s'appuyant sur les résultats remises en cause depuis les années 1980 par la théorie des marchés contestables, un véritable défi est lancé au paradigme SCP.

\subsubsection{La théorie des marchés contestables}

La notion de barrières à l'entrée est l'un des concepts fondamentaux de l'économie industrielle et un fondement majeur de l'approche SCP. En effet, l'absence des barrières à l'entrée réduit également la capacité des 
entreprises à maintenir des solutions de monopole du fait de la concurrence potentielle. Si les concurrents potentiels ont la possibilité d'obtenir des marges positives, ils peuvent alors décider d'entrer sur le marché. Dans ce cas, les firmes installées ont le choix entre l'abaissement des prix au niveau qui rend l'entrée non rentable pour les concurrents potentiels ou alors subir la concurrence. Les firmes installées sur le marché peuvent également instaurer d'autres barrières à l'entrée avec une protection spécifique ou avec une réglementation des pouvoirs publics, imposant des règles d'accès. J.S.Bain ( 1956) a été le premier auteur qui a révélé l'importance stratégique des barrières à l'entrée dans la détermination des structures et des performances des marchés, selon lui, la définition des barrières à l'entrée devient plus précise et complète en se focalisant sur la question du pouvoir de marché ou de monopole. Cette notion de pouvoir de marché exprime la capacité des firmes établies à fixer leurs prix de vente au-dessus du seuil des prix d'équilibre dans un marché parfaitement concurrentiel. Même principe repris également par par F.M.Scherer et D.Ross (1970), ces derniers ont rajouté une quatrième composante au paradigme SCP, celle des caractéristiques de l'offre et de la demande, elles regroupent selon eux, l'ensemble des conditions de base de l'industrie, celles-ci occupent une place importante dans l'analyse industrielle. La structure du marché est déterminée par le nombre d'offreurs et de demandeurs, la différenciation des produits, les barrières à l'entrée, l'intégration verticale et par la stratégie des coûts, sont toutes des variables qui influencent les choix stratégiques des firmes. Ces facteurs de base sont guidés normalement par les politiques de fixation des prix, de produit, de publicité, de recherche et d'innovation, qui par la suite déterminent la performance (Scherer \& Ross, 1970).

L'approche structuraliste de J.S.Bain et de F.M.Scherer a été critiquée par plusieurs économistes notamment ceux de l'école de Chicago à l'instar de G.J.Stigler (1964) et Y.Brozen ( 1971), selon ces derniers auteurs, la relation entre la structure et la performance d'une industrie doit être inversée. En effet, un choix comportemental peut bien affecter la structure de l'industrie et simultanément, la performance affecte le comportement mais aussi la structure ((Stigler, 1964) ; (Brozen, 1971)). Par ailleurs J.Tirole (1988) et d'autres auteurs de l'école française de la Nouvelle Economie Industrielle critiquent l'absence de la prise en considération de l'intervention des pouvoirs publics dans le paradigme SCP. Ils considèrent ainsi qu'il est important d'observer les actions des pouvoirs publics comme un élément affectant les conditions de base d'une industrie et sa structure. Telle intervention se pratique par la réglementation et par la politique des prix.

La limite fondamentale de la théorie des marchés contestables est l'absence des réactions des firmes installées à l'entrée des nouveaux concurrents. Ce qui exclut les comportements concurrentiels. Pour développer 
cette théorie, une nouvelle approche a vu le jour pour combler les lacunes observées, c'est la théorie des jeux.

\subsubsection{La théorie des jeux}

Avec l'essor de la théorie des jeux, l'économie industrielle va connaître un profond renouvellement dans les années 1980-1990. Cette dernière va devenir la référence des économistes industriels, elle va servir à la modélisation des comportements et des interactions des entreprises sur les marchés en employant des propositions testables. Les techniques économétriques vont permettre de mieux estimer la nature des relations entre les structures, les comportements et les performances. Cette nouvelle approche offre aussi une relecture stratégique du paradigme SCP, un prolongement au niveau d'analyse, l'idée est que les structures et les comportements pourront avoir une interaction dans les deux sens. Ainsi la structure d'un marché peut dépendre également des stratégies des entreprises.

L'existence d'un petit nombre d'acteurs industriels en concurrence et la présence de règles du jeu parfois élaborées par les régulateurs font des télécommunications un champ d'application privilégié pour la théorie des jeux. Les directions générales ou de la stratégie des grands opérateurs des télécommunications, ainsi que les autorités de régulation, utilisent la théorie des jeux pour stimuler les comportements stratégiques dans les cas d'ouverture de marché ou de rapprochements d'acteurs.

Malgré les critiques adressées au paradigme SCP, ce modèle a été et reste le cadre de référence basique et l'un des modèles les plus utilisés dans les études de la concurrence en économie industrielle.

\subsection{Cadre empirique}

En parallèle des avancées de la théorie économique, des thématiques diversifiées ont été traitées en rapport au sujet des télécommunications, on peut évoquer celles relatives à la réglementation et à la concurrence (Encaoua, 1986), de l'économie des réseaux de télécommunications (Curien \& Gensollen, 1987), à l'accès aux infrastructures par les opérateurs alternatifs au regard de leur détention par l'opérateur historique et l'interopérabilité des réseaux (Pénard \& Thirion, 2007) à la problématique des droits exclusifs détenus par la plupart des opérateurs historiques et à l'abus de position dominante et la justification de la régulation (Boylaud \& Nicoleti, 2011), à l'évolution des marchés de la téléphonie mobile (Gruber \& Verboven, 2001). La plupart de ces études sont inscrites dans le champ disciplinaire de l'économie et/ou du droit, avec les instruments des sciences économiques et politiques. Ainsi, les approches stratégistes et concurrentielles des opérateurs de télécommunications sous l'angle de l'économie industrielle dans un contexte de libéralisation sont peu abordées, surtout avec la mobilisation du 
paradigme SCP comme cadre d'analyse. La présentation de volet empirique se contentera donc aux études mobilisant l'aspect de l'ouverture à la concurrence et/ou les études portant sur le paradigme SCP sans pour autant allier la régulation du secteur dans ces études.

On commence par l'étude de la relation entre la structure du marché de la téléphonie mobile et la performance des firmes du secteur, de nombreuses études empiriques ont examiné cette causalité. P.M.Parker et L.H.Röller étudient aux USA durant la période (1984-1988) l'impact de l'entrée d'un nouveau concurrent sur les prix et sur les comportements du duopole américain. L'objectif principal de cette étude est de tester le niveau concurrentiel du marché de la téléphonie mobile. Ces auteurs ont exploité les variables de la demande et de la structure, les prix des services, les prix de la production et les prix des facteurs d'entrée. Les résultats retenus ont confirmé l'impact de la libéralisation du secteur de la téléphonie mobile sur la baisse des prix des services mobiles, cette baisse a engendré par conséquent une augmentation des chiffres d'affaires et donc ils ont déduit une relation positive entre la structure du marché de la téléphonie mobile et la performance des entreprises (Parker \& Röller, 1997).

Une autre étude américaine porte sur la concurrence de la téléphonie mobile, développée par H.S.Fullerton en 1998. Les résultats de cette étude ont montré que la structure du marché n'est pas le seul élément qui influence les comportements des firmes et par la suite sur la performance. Il a indiqué que le duopole qui existait à cette phase a provoqué la concurrence des prix malgré la différence au niveau de la performance d'un marché à un autre (Fullerton, 1998).

L'industrie européenne des télécommunications mobiles faisait l'objet d'une étude menée par Z.Fernandez et B.Usero en 2009. En analysant les comportements des opérateurs historiques (leaders) et les autres opérateurs concurrents (followers), les auteurs ont pu conclure la diversité des comportements des opérateurs, l'objectif de l'opérateur historique est d'améliorer ses parts de marché donc, il insiste sur les stratégies de différenciation tandis que les opérateurs concurrents s'orientent plutôt vers les prix en essayant de réduire l'écart de la position dominante des opérateurs leaders, (Fernandez \& Usero, 2009).

Une autre étude de L.Grzybowski et P.Pareira élaborée en 2007 en Portugal. Cette étude analyse les effets de l'entrée d'un troisième opérateur et la libéralisation de la téléphonie fixe sur la performance de la téléphonie mobile, plus précisément sur les coûts et la concurrence entre les opérateurs de segment mobile en Portugal. Les résultats étaient significatifs, le changement de la structure du marché avec l'arrivée d'Optimus a eu un impact positif sur la performance du marché de la téléphonie mobile au Portugal (une baisse des coûts et une concurrence accentuée), tandis que la libéralisation de 
segment fixe n’avait pas un impact réel sur les coûts (Grzybowski \& Pareira, 2007).

En Italie, un article de L.Cricelli et al. apparu en 2002, 1'étude porte sur l'impact d'un nouvel opérateur sur les parts de marchés et les prix. Le marché mobile italien à l'époque était un marché oligopole de trois opérateurs. L'étude consiste à mesurer l'effet d'entrée d'un quatrième opérateur sur le marché mobile italien. Les auteurs ont prouvé la baisse importante des prix après la hausse de la concurrence et la multitude d'offre de services liée à la convergence entre la téléphonie fixe et mobile. Les chercheurs ont montré que les coûts d'interconnexions, les prix des appels et les quantités offertes ont été les principaux facteurs de maximisation des profits chez les opérateurs, (Cricelli, Massimo, \& Levialdi, 2002).

Pour les études régionales, une étude mobilise le paradigme SCP publiée par les auteurs A.Ait Bari et A.Lahoucine (2016). Les deux chercheurs ont testé un modèle empirique basée sur des données de panel sur la période (2004-2014) pour vérifier l'impact de la structure de l'industrie de la téléphonie mobile au Maghreb sur la performance des opérateurs en place. Les variables les plus pertinents retenues pour cette étude sont: Le profit, la concentration, l'effet de la demande et de l'offre. Ces résultats montrent que $64,74 \%$ de la variation du profit de l'industrie de la téléphonie mobile au Maghreb est expliquée par des variables liées à la structure du marché. De même pour la demande, lorsque le nombre des abonnés aux services de la téléphonie mobile par cent personnes augmente, le profit de cette industrie s'accroit. Comme conclusion, les auteurs ont vérifié l'influence positive de la concentration sur la performance et la rentabilité des opérateurs maghrébins, ils ont expliqué ce résultat par l'importance des barrières à l'entrée qui limite le nombre des concurrents (Ait Bari \& Lahoucine, 2016).

Pour les études purement domestiques sur le marché marocain, il y’a lieu de citer l'unique rapport de conseil de la concurrence publié en 2011. Ce rapport présente les résultats d'une étude relative à la concurrence dans le secteur de la téléphonie mobile en employant les principes du paradigme SCP. Cette étude renforce l'action de la régulation pour promouvoir la concurrence. Elle met l'accent également sur la forte croissance de la demande de communication enregistrée entre 2000-2010, cette demande a retardé la confrontation entre les concurrents mais malgré cette donne, les prix n'ont pas connu une baisse notable. Selon l'analyse de la structure du marché mobile des télécommunications, l'existence des barrières à l'entrée et la forte concentration du marché sont des éléments parmi d'autres qui défavorisent la concurrence intense dans ce secteur. Pour intensifier la concurrence, le partage des infrastructures, le dégroupage, le règlement des tarifs d'interconnexion deviennent une nécessité (Conseil de la concurrence, 2011). 


\section{Conclusion}

A travers cette revue de littérature, nous avons abordé la thématique de la concurrence d'un marché industriel qui est le marché de la téléphonie mobile qui s'inscrit prioritairement dans le champ théorique de l'économie industrielle, discipline de la microéconomie qui analyse les comportements et les stratégies des entreprises et de la concurrence sur les marchés (Carlton \& Perloff, 2008). La méthodologie de l'économie industrielle que nous avons proposée ainsi que, les études théoriques et empiriques présentées brièvement ont toutes aboutit aux résultats plus ou moins similaires aux enseignements théoriques du paradigme SCP. Pour le marché marocain de la téléphonie mobile, l'ouverture à la concurrence a imposé des réformes réglementaires et institutionnelles importantes, le pays a aujourd'hui une opportunité pour accélérer son développement et renforcer sa compétitivité. La mise en place d'un environnement plus propice au développement des secteurs émergents comme celui de la téléphonie mobile prendra donc une place de choix dans la société de demain et leur succès conditionnera tous les secteurs du Maroc. Or, les études scientifiques et académiques abordant l'économie industrielle comme outil d'analyse restent générales et rares, c'est fort de ce constat et sur la base de cette préoccupation que nous avons bâti notre objet de recherche. La construction de modèle conceptuel de la recherche ainsi que les résultats de l'étude empirique de notre travail sont en cours de réalisation et seront publiés prochainement. 


\section{References:}

1. ANRT (2001). Rapport annuel. Agence nationale de réglementation des télécommunications.

2. Ait Bari, A. \& Lahoucine, A. (2016). Impact de la structure du marché sur la performance de l'industrie de la téléphonie mobile au Maghreb. Revue Economie, Gestion et Société.

3. Bain, J.S. (1956). Barriers to new competition. Cambridge, Harvard University Press.

4. Baumol, W.J. P. (1982). Contestable Markets and the theory of industry structure. New York: Harcourt.

5. Boylaud \& Nicoleti (2001). Le secteur des télécommunications, structure du marché et performance. Revue économique de l'OCDE $N^{\circ} 32$.

6. Brozen, Y. (1971). Concentration and Rates of Return Revisited. Journal of Law and Economics, vol.14, 351-370.

7. Carlton, J. \& Perloff, D. (1988). Economie Industrielle. Edition DeBoeck.

8. Catelin, C. \& Chatelin, C. (2001). Privatisation, gouvernement d'entreprise et processus décisionnel : une interprétation de la dynamique organisationnelle à travers le cas France Télécom. Finance Contrôle Stratégie, vol.4, $N^{\circ} 2,63-90$.

9. Chairman, W.E. \& William, E.C. (1999). Connecting the word. Washington: Federal Communications Commission. (FCC).

10. Chatelin, C. (2002). Quels enjeux théoriques et méthodologiques de la théorie de la gouvernance partenariale ? La privatisation comme illustration. Laboratoire Orléanais de Gestion, Document de recherche $N^{\circ} 2002-05$.

11. Conseil de la Concurrence (2011). Etude de la concurrentiabilité du marché de la téléphonie mobile au Maroc.

12. Cricelli, L. (2002). The impact of competition in the italian mobile telecommunications market. Net work and Spatial Economics,vol.2 $N^{\circ} 3,239-253$.

13. Curien, N. (2000). Economie des réseaux. Paris: La Découverte.

14. Curien, N. Muet, P. (2004). La société de l'information. Paris: Conseil d'analyse économique, La Documentation française.

15. Dang Nguyen, D. (2000). Economie des télécommunications et de l'Internet. Paris: Economica.

16. Demsetz, H. (1973). Industrial structure, market rivalry and public policy. Journal of Law and Economics, vol.16, 1-9.

17. Fernandez, Z. (2009). Competitive behavior in the European mobile telecommunications industry: Pionneers vs Followers. Telecommunications Policy, vol.33 No7, 339-347. 
18. Fullerton, H. (1998). Duopoly and competition,the case of american cellular telephone. Telecommunications Policy,vol.22 N7, 593- 607.

19. Gentzoglanis,A., Sundberg,N. \& Shorr, S. (2001). Une réglementation efficace. Etude de cas : le Maroc. Genève: l'Union Internationale des Télécommunications.

20. Gruber, H. \& Verboven, F. (2001). The evolution of markets under entry and standards regulation, the case of global mobile telecommunication. International Journal of Industrial Organization $N^{\circ} 19(7), 1189-1212$.

21. Grzybowski, L., \& Pareira, P. (2007). Merger simulation in mobile telephony in Portugal. Review of industrial organisation,vol.3 $\mathrm{N}^{\circ} 3$, 205- 220.

22. Hassani Allaf, Z. (2017). Libéralisation et régulation des télécommunications au Maroc. Revue droit et entreprise $N^{\circ} 14,216$ 234.

23. Hassani Allaf, Z. \& Drissi Bakhkhat, M. (2018). Libéralisation du marché de la téléphonie mobile au Maroc. Revue des études multidisciplinaires en sciences économiques et sociales $N^{\circ} 8,141-161$.

24. Hibou, B. \& Tozy, M. (2002). De la friture sur la ligne des réformes: Libéralisation des télécommunications au Maroc. Critique internationale $N^{\circ} 14,91-118$.

25. Intven, H. (2000). Vue d'ensemble de la réglementation des télécommunications. La Banque mondiale.

26. Lien, D. \& Peng, Y. (2001). Competition and Production Efficiency Telecommunications in 24 OCED's Countries: Information Economics and Policy,vol.13, 51-76.

27. Lorenzi, J-H. \& Didier. (2002). Le secteur des télécommunications entre monopole et oligopole $\mathrm{N}^{\circ} 158$.

28. Mason, E. S. (1957). Economic concentration and the monopoly problem. Harvard University Press.

29. Mezouaghi, M. (2005). Libéralisation des services de télécommunications au Maghreb. AFD (agence française de développement). Note et documents $N^{\circ} 23$.

30. Parker, P. M. \& Röller, L. H. (1997). Collusive conduct in duopolies: multimarket contact and crossownership in the mobile telephone industry. The RAND Journal of Economics, 304-322.

31. Sherer, F.M. \& Ross (1970). Industrial Market Structure and Economic Performance. Chicago: Rand Mc Nally.

32. Smith, A. (2011). Le changement dans la régulation de la globalisation : de l'Economie politique internationale au travail politique mondial. Communication à la section thématique $N^{\circ} 2$ (Penser le changement 
international) du congrès de l'Association française de la science politique, Strasbourg.

33. Stigler, G. (1964). A theory of Oligopoly. The journal of Political Economy,vol.72, Issue 1.

34. Tirole, J. (1995). Théorie de l'organisation industrielle. Edition Economica.

35. Tirole, J. \& Laffont, J. (2000). Competition in Telecommunications. Cambridge: MIT Press.

36. UIT (2008). Partager l'infrastructure pour développer la connectivité. Nouvelles de l'UIT Nº2.

37. Wallsten, S. (2001). An Econometric Analysis of Telecom Competition, Privatization and Regulation in Africa and Latin America. The Journal of Industrial Economics.vol.49, 51-76

38. Word Bank (2004). Morocco, developing competition in telecommunication. Global information and communication technologies department. 